\title{
Impact of pulsed jet actuators on aircraft mass and fuel consumption
}

\author{
Francesco G. A. Bertels ${ }^{1} \cdot$ Reinier van Dijk ${ }^{1} \cdot$ Reno Elmendorp ${ }^{1} \cdot$ \\ Roelof $\operatorname{Vos}^{1}$
}

Received: 21 October 2015/Revised: 16 June 2016/Accepted: 11 July 2016/Published online: 11 August 2016

(c) The Author(s) 2016. This article is published with open access at Springerlink.com

\begin{abstract}
Pulsed jet actuators (PJAs) are one of the candidate technologies to be integrated in Fowler flaps to increase the maximum lift coefficient of transport aircraft in the landing configuration. The total system consists of the actuators plus sensors, a piping system to supply pressurized air and a (redundant) power and communication system to provide actuator control. In this paper, it is investigated what increase in the maximum lift coefficient is required to justify the added weight and power off-takes that accompany the integration of pulsed jet actuators. This is done by making an automated design process for the overall aircraft, the piping assembly system, and the electrical wiring interconnection system. These last two subsystems rely on KBE techniques that automate dimensioning and performance evaluation. A test case is specified that encompasses the design of a typical single-aisle midrange aircraft with and without the PJA system installed. It is concluded that the introduction of the PJA system requires at least an increase in maximum lift coefficient of 0.2 to justify the increase in system mass and power offtakes. Furthermore, it is shown that if the maximum lift coefficient increases with 0.4 , only small reductions in maximum take-off weight $(-0.3 \%)$ and operating empty weight $(-0.6 \%)$ can be expected, while the total fuel burn remains virtually constant.
\end{abstract}

This paper is based on a presentation at the CEAS Air \& Space Conference 2015, September 7-11, Delft, The Netherlands.

Roelof Vos

r.vos@tudelft.nl

1 Delft University of Technology, Faculty of Aerospace Engineering, Kluyverweg 1, 2629 HS Delft, The Netherlands
Keywords Pulsed jet actuators · Knowledge-based engineering · Aircraft design · Aircraft systems . Technology assessment

\section{Introduction}

Active flow control (AFC) is a subject that has gained considerable interest in the past years as a solution to the never-ending demand of further improving the efficiency of aircraft. However, the number of instances where AFC has successfully transitioned from a laboratory prototype to a real-world aeronautical application is small [1-6]. One of the most important applications of AFC is the delay of separation to increase the maximum lift coefficient of an aircraft with high-lift devices. Even with modern simulation techniques using high-fidelity computational fluid dynamics (CFD), it is difficult to give a reliable prediction of the effect of AFC on the maximum lift coefficient of a full-scale transport aircraft that employs slotted high-lift devices. Theoretical studies on a two-dimensional wing with slat and flap demonstrated a possible increase of $c_{l \max }$ of 0.7 [7]. Even though the benefits are difficult to quantify, the penalties in terms of power consumption and weight addition can be estimated using knowledge-based design principles and first-order analysis techniques. In this paper, the effect of a pneumatic pulsed jet actuator on the fuel weight and maximum take-off weight of a mid-range, highsubsonic jet transport is considered under the assumption of a predefined increase in maximum lift coefficient. It is investigated what increase in the maximum lift coefficient is required to justify the added weight and power off-takes that accompany the integration of pulsed jet actuators. This reverse approach to the assessment of pulsed jet actuators does not require an expensive and unreliable $\mathrm{CFD}$ 


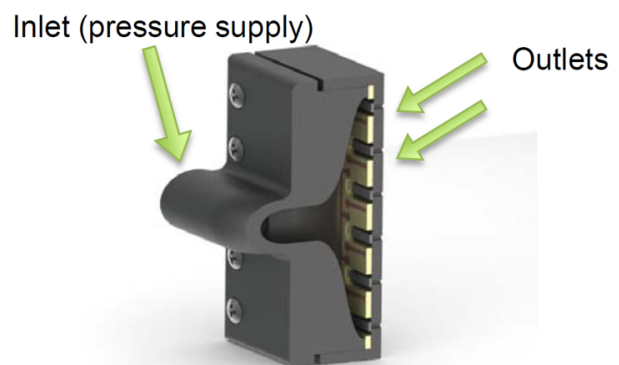

(a) Cross-section schematic view

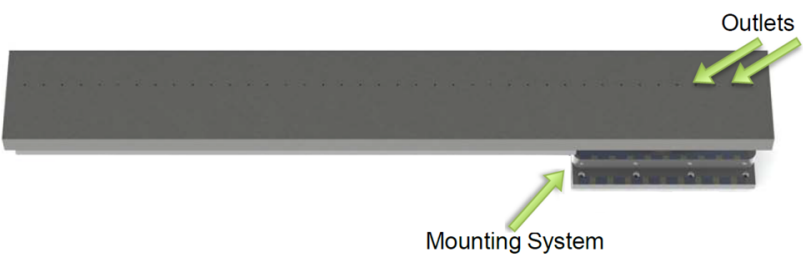

(b) Assembly of 4 standard strips containing 40 outlets.

Fig. 1 Fraunhofer pulsed jet actuators (PJAs)[8]

investigation and can give a first indication of the feasibility of such a system.

For this study, the pulsed jet actuators (PJA) under development by Fraunhofer ENAS are used [8]. These actuators rely on a continuous mass flow of compressed air and use piezoceramic valves to achieve the pulsation of the jet stream. Figure 1a depicts a schematic picture of one of the actuators consisting of 6 individual orifices spaced $1 \mathrm{~cm}$ apart. Figure $1 \mathrm{~b}$ depicts how four standard actuators of ten orifices are mounted next to each other to form a $40-\mathrm{cm}$ long strip containing 40 orifices. The Fraunhofer PJA concept is based on single piezoelectric elements that are able to switch every single orifice individually. Each actuator consists of ten orifices and needs to be connected to an air supply and a signal generator to operate.

This strip of actuators is to be embedded on the top surface of a Fowler flap to form a row of orifices that spans the complete flap. Although multiple rows of actuators are possible, the current research is limited to a single row of actuator orifices. A set of pressure sensors behind the actuators sense the state of the airflow over the flap. Based on this information, the actuators can be commanded to provide pulsated blowing. A pressurized air pipe runs parallel to the actuators to provide compressed air, while power and communication cables are required to actuate the piezoceramic valves inside the actuators. A schematic overview of the embedded system in the flap can be seen in Fig. 2.

The Fraunhofer PJAs are embedded as standardized subassemblies of four actuators (see Fig. 1b). Each actuator requires a mass flow of $8.5 \mathrm{~g} / \mathrm{s}$ at a maximum temperature of $100{ }^{\circ} \mathrm{C}$. To provide a sufficiently strong jet, the pressure differential over the orifice should be 600 mbar. The

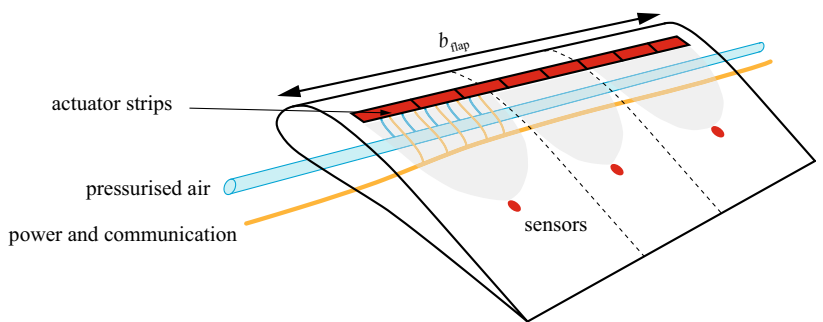

Fig. 2 Schematic representation of PJA implementation in a flap

piezoceramic valves inside the actuator require a sinusoidal signal of $500 \mathrm{~Hz}$ with a voltage of $230 \mathrm{~V}$ (root mean square voltage). Per 4 actuators a current of $100 \mathrm{~mA}$ is required. This signal is delivered by a DC/DC converter in combination with a signal generator. The system is subsequently connected to the $230 \mathrm{~V}$ on-board alternating current electrical system. A high-voltage, applicationspecific integrated circuit (HV-ASIC) is added to control the functioning of the actuator system. Figure 3 shows the interconnections of all PJA components, their specifications and dimensions.

The goal of this paper is to rationalize the overall performance impact of these PJAs for a mid-range aircraft. The specifications that are presented above function as the starting point of the system design. Since there is no reliable data on the aerodynamic benefits of the actuators, an assumption has to be made on the increase in maximum lift coefficient that they can bring to the aircraft. In the next section, the methodology for the performance assessment of the PJAs will be detailed. Subsequently, the results of this investigation will be presented and discussed. Finally, it will be concluded what increase in maximum lift coefficient is required to justify the increase in system weight and power consumption that accompany the introduction of PJAs in Fowler flaps. This research was conducted within the Smart Fixed Wing Aircraft (SFWA) framework as part of the Clean Sky 1 program.

\section{Design and assessment methodology}

Figure 4 shows a flow diagram of the technology assessment approach that is taken. First, a set of top-level mission requirements is specified. These requirements dictate the overall aircraft configuration and prescribe range, payload weight, number of passengers, and maximum take-off and landing distance. In the subsequent level, an automated conceptual design process for transport aircraft synthesizes an aircraft based on these requirements according to the process documented in Elmendorp et al. [9] The aircraft design tool uses classical handbook methods to compute a Class II design. Refined, semi-analytical estimations for fuselage weight, wing weight, engine performance, landing 


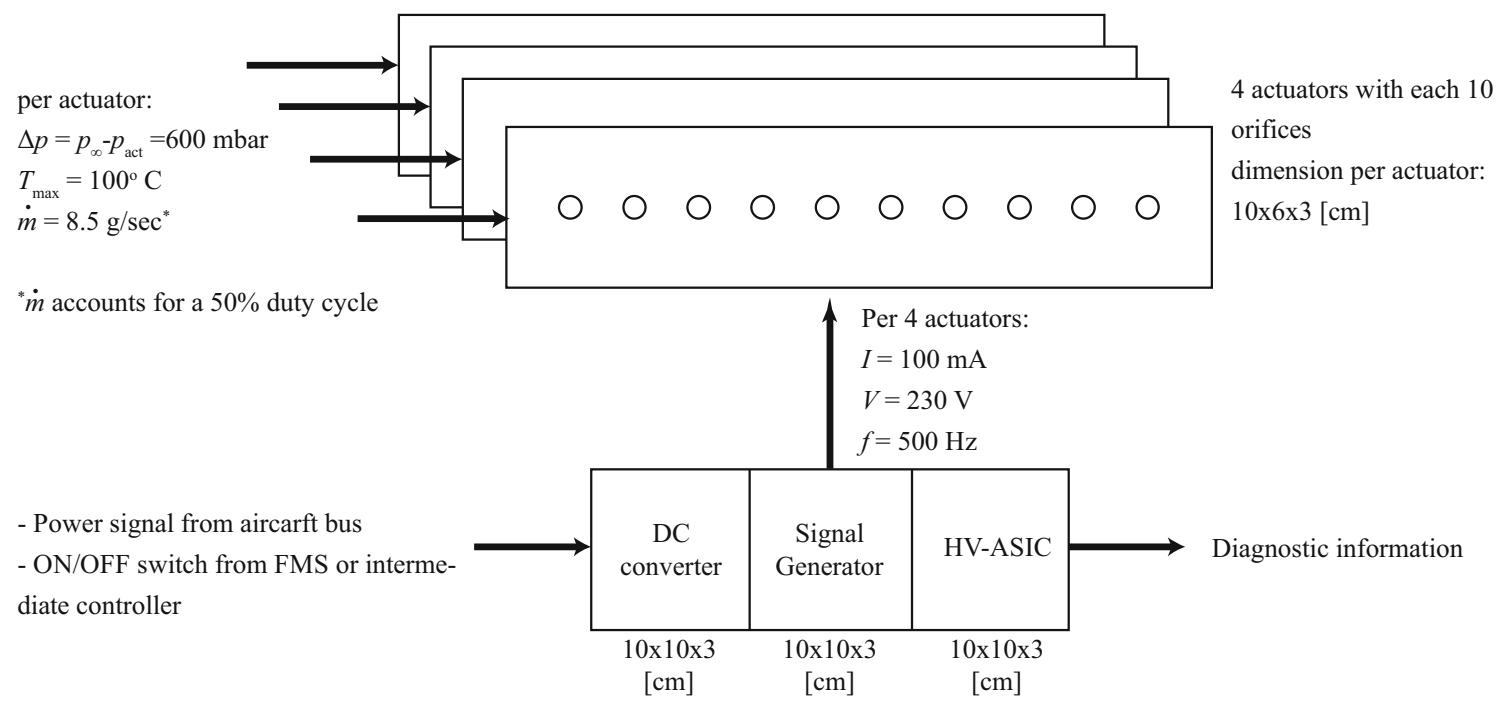

Fig. 3 Schematic representation of PJA system components, dimensions and specifications

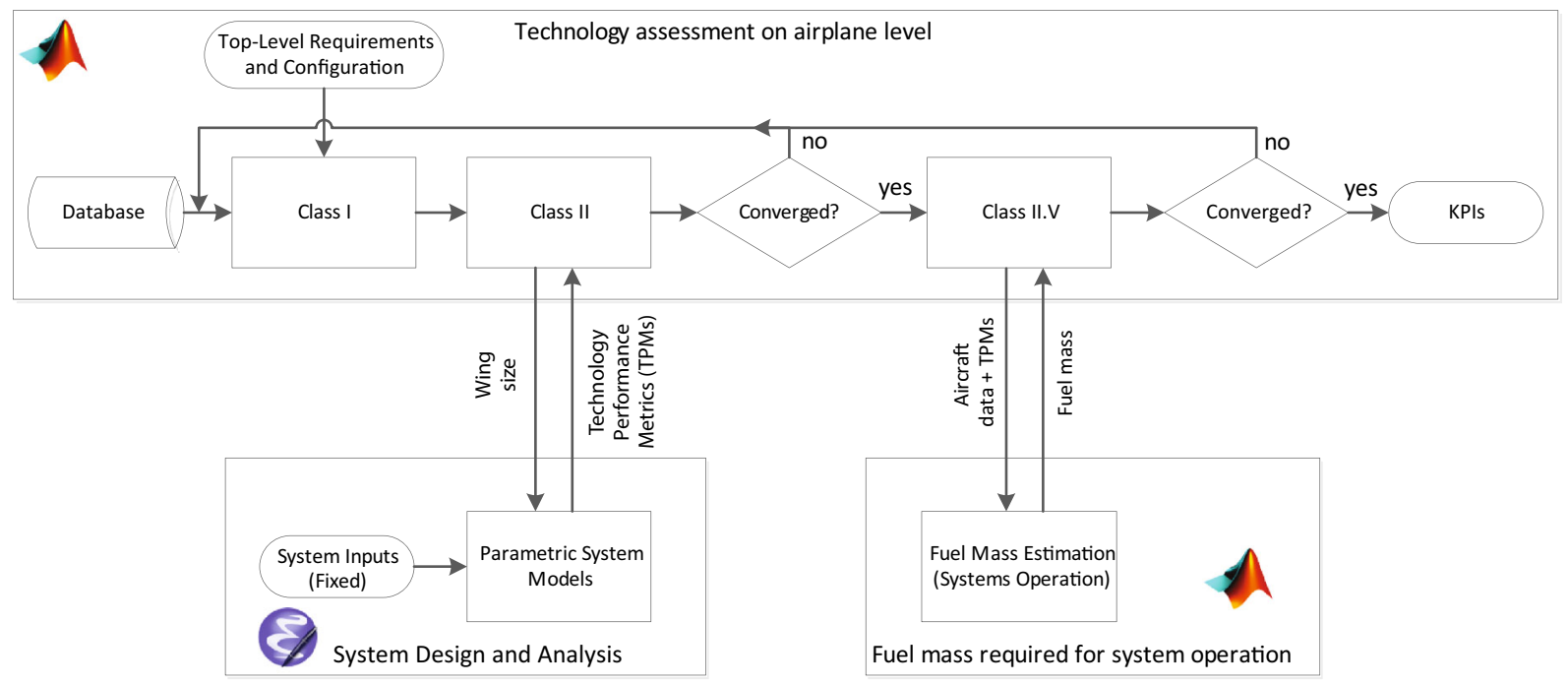

Fig. 4 Methodology to predict technology impact on aircraft level

gear weight, and fuel consumption are subsequently used to further refine the overall performance estimations. These methods are called Class II.V methods. Using the Class II.V methods, the performance indicators such as fuel consumption and maximum take-off weight are estimated. Below this layer of conceptual design methods, a preliminary design layer is located which contains an automated design methodology for the system architecture (left) and an estimation of the system fuel consumption (right). These will be further elaborated in this section.

To compute the weight and power consumption of the PJA system, the full system architecture inside the aircraft is automatically designed. Apart from positioning the actuators, this consists of pneumatic piping with optional pump and an electrical wiring system to power and control the actuators. Both systems are automatically designed using design rules that have been captured from industry practice. The design software merely uses the location of the actuators, power centers, and pressure source to automatically determine the shortest route for piping and wiring, thereby conforming to using predefined paths and levels of redundancy. Subsequently, the weight of the system is estimated and the required power (both electrical and pneumatic) is computed.

In Fig. 5 a schematic overview of the system architectures is presented. The green lines indicate the pneumatic pipes, while the red lines indicate the possible path of the Electrical Wire and Communication System (EWIS). It can be seen that the wiring architecture in the fuselage consists of two main paths that run longitudinally on either side of the fuselage below the main passenger deck. Several cross paths exist to interconnect these two main paths. In the 


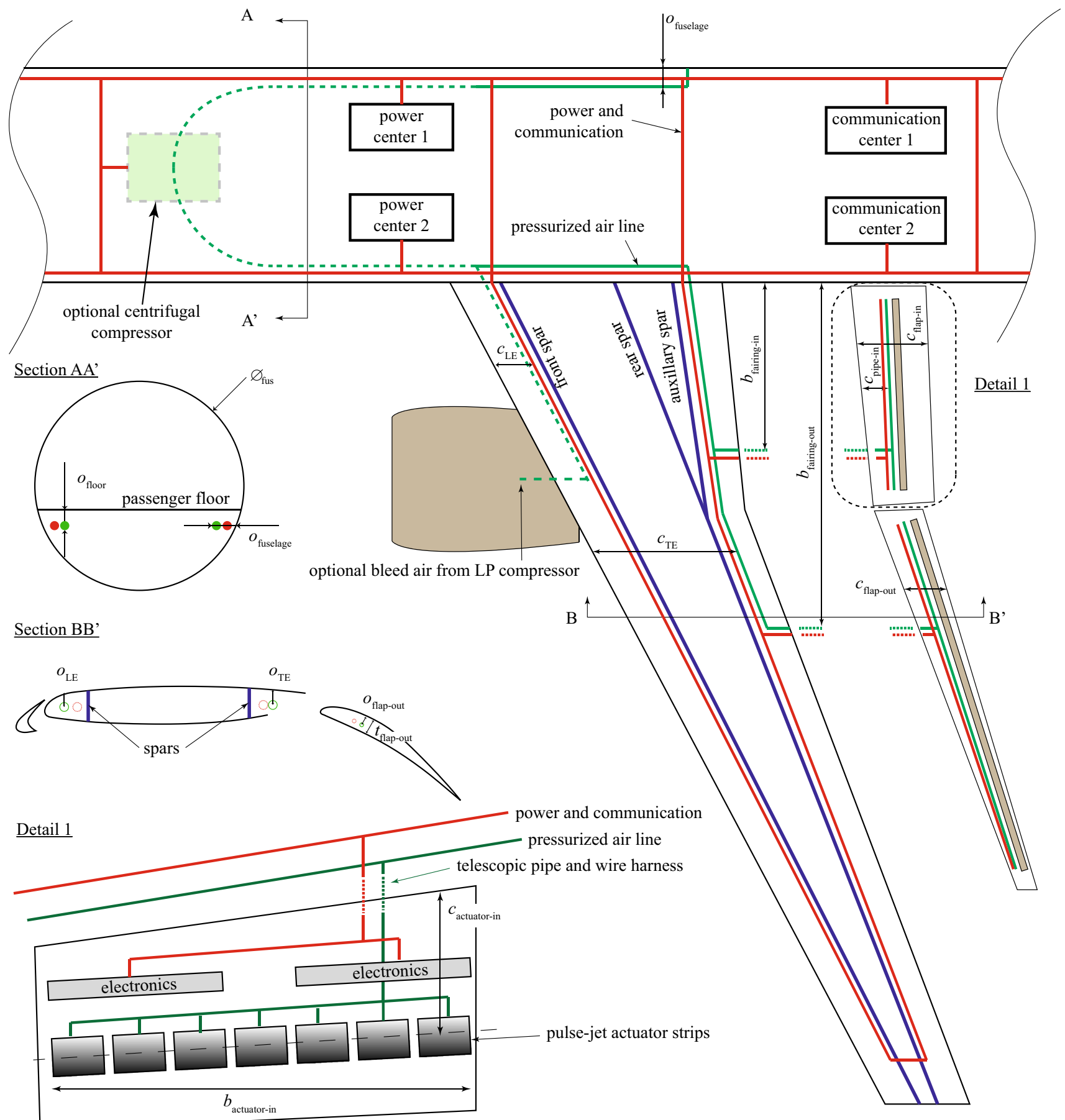

Fig. 5 Overview of EWIS and pnuematic piping to support pulsed jet actuators on Fowler flaps. Note that pressurized air comes either from the low-pressure compressor of the engine or a separate centrifugal compressor powered by an electro motor

wing, there are two main paths: one ahead of the front spar and one behind the rear spar. Due to the fuel tank in between the two spars, there is only a single cross connection that connects these two paths near the tip of the wing. The pneumatic piping connects the clients (in this case the PJAs) directly to a source of compressed air. Two source options are available: either the air is bled off from the low-pressure compressor of the engine, or an electrically powered on-board compressor is used. The following subsections will further detail the design and analysis of the piping assembly and EWIS system to support the PJA operation. 


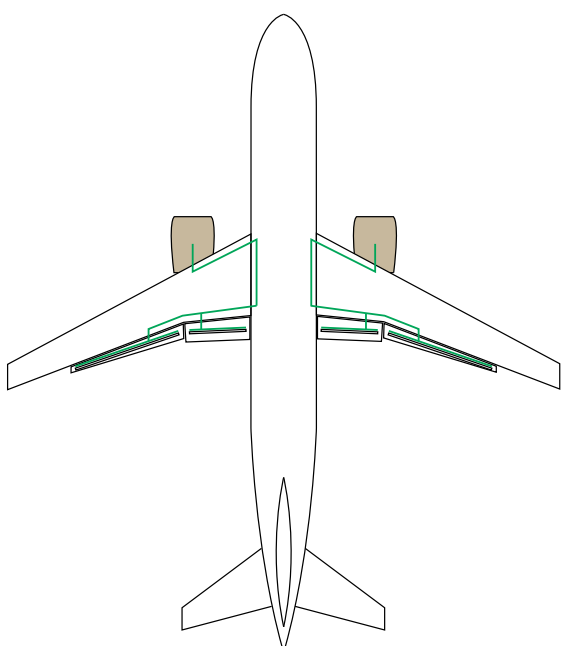

(a) Wing-mounted engines and bleed air

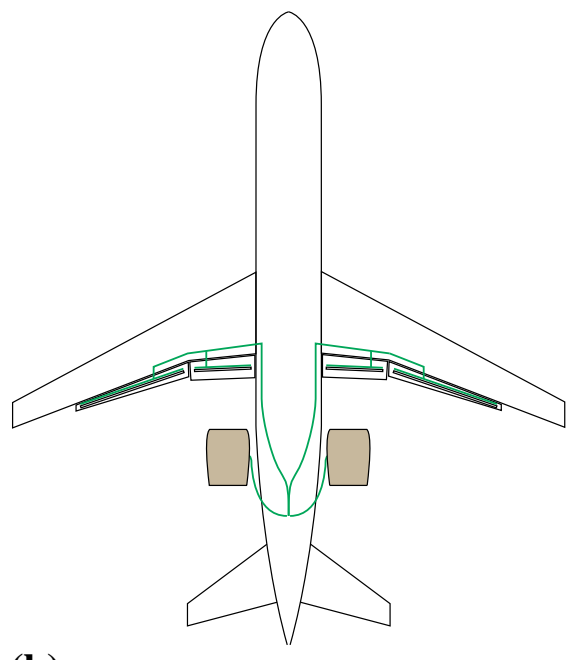

(b) Fuselage-mounted engines and bleed air

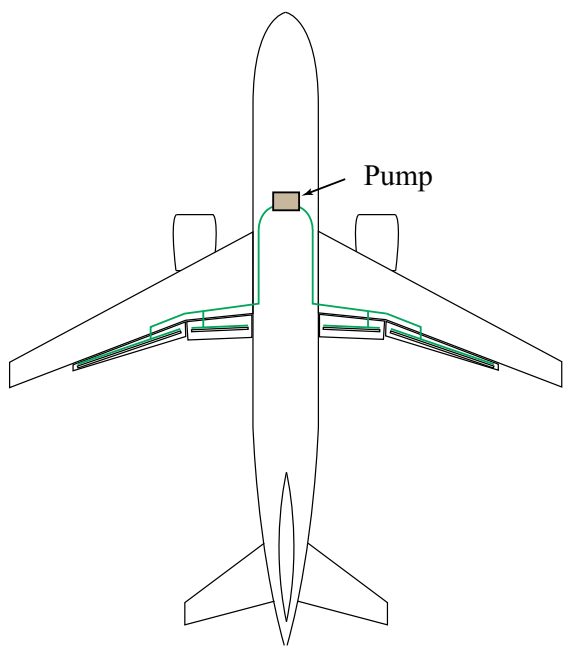

(c) Wing-mounted engines and separate pump

Fig. 6 Examples of piping assembly configuration

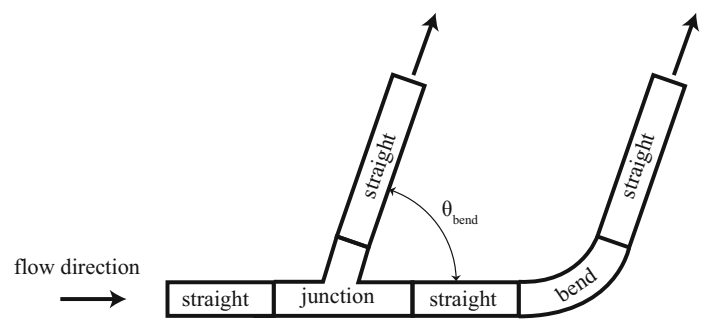

(a) Building blocks to design the pneumatic piping
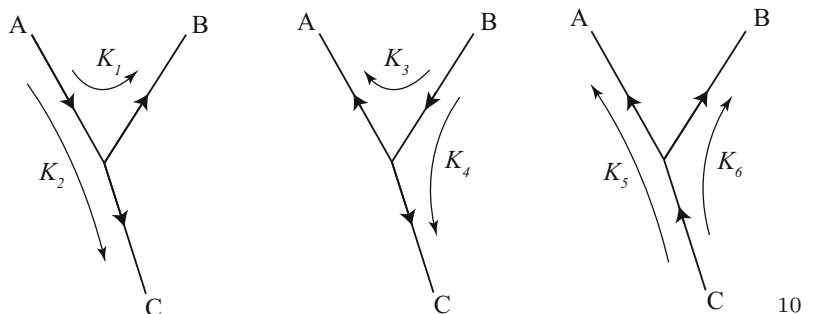

(b) Possible flow types and losses for a three-pipe junction with separating flow

Fig. 7 Sketches to support pipe analysis

\subsection{Piping assembly design and analysis method}

In the case study presented in this paper, three different global architectures are modeled: bleed air from engines mounted on the wing, bleed air from engines mounted on the fuselage, or the use of a centrifugal compressor to compress the free stream air. Figure 6 depicts a top view of these three different architectures as implemented in the design tool.

The piping assembly is designed by first determining the pipe paths on the aircraft. These paths are constructed using main duct paths and connecting paths. The routing is obtained automatically by specifying the outer shape of the wing, flaps, engines and fuselage in addition to a number of user inputs. These inputs allow the user to parametrically define the complete piping path. These inputs are depicted in Fig. 5 by means of arrows (i.e. $C_{\text {flap-in }}$ is the chordwise distance of the pipe from the leading edge of the inboard flap). In addition to these inputs, the user needs to specify the pipe material, the wall thickness (typically $1 \mathrm{~mm}$ ), the diameter of the actuator inlet, and $\theta_{\text {bend }}$, i.e. the angle at which junctions and connecting segments branch out (see Fig. 7a). Because the program is not capable of properly modeling the junction geometry and the additional material that is typically added to solder all the parts together, a mass correction factor is identified: $1.78 .^{1}$ This value is multiplied to the original mass estimation of the pipe assembly.

1 To estimate this correction factor the program was used to model an existing bleed air piping component. The estimated weight was compared to the measured weight resulting in this value. 
Apart from the piping geometry generation, the tool also analyzes the flow conditions in the whole piping system to determine the initial flow conditions necessary to be delivered by the engines or the pump. Since this tool is meant to be used in the conceptual design phase with many design iterations, the flow analysis is performed using a low-order method. For each of the three different piping blocks, depicted in Fig. 7a, a methodology is presented to estimate the upstream flow conditions. The analysis starts downstream where the actuators are connected to the piping assembly and the desired flow conditions are known from the actuator specifications. Subsequently, the flow condition is estimated all the way upstream to the source of the pressurized air.

The pressure drop for a straight pipe with a constant diameter is primarily derived from internal wall friction. If isentropic flow is assumed, the Darcy-Weisbach equation can be used to find the approximate pressure drop [11]:

$$
\Delta p=\frac{\rho f L u^{2}}{2 D_{h}}
$$

where $f$ is the friction factor, $L$ the length of the pipe and $D_{h}=4 S / P$ the hydraulic diameter where $S$ is the crosssectional area and $P$ the wetted perimeter. The friction factor can be determined using the Colebrook equation:

$$
\frac{1}{\sqrt{f}}=-2 \log _{10}\left(\frac{\epsilon / \mathrm{D}}{3.7}+\frac{2.51}{\operatorname{Re} \sqrt{\mathrm{f}}}\right)
$$

with Re being the Reynolds number and $\epsilon / D$ being the relative roughness, i.e., the ratio of the mean roughness height and the pipe diameter [11]. Typical values for aluminum pipes are $0.001-0.002 \times 10^{-3} \mathrm{~m}[12]$.

Bends in pneumatic systems lead to additional pressure losses when compared to straight pipes. These losses can be accounted for using an equivalent length $\left(L_{e}\right)$ which substitutes $L$ in Eq. 1 . The pressure drop through a $90^{\circ}$ bend is estimated using the following equation [13]:

$$
\begin{aligned}
\left(\frac{L_{e}}{D}\right)_{90^{\circ}}= & 19.833\left[0.003625+0.038 \sqrt{\frac{2 R_{c}}{D \sqrt{\mathrm{Re}}}}\right] \\
& \times \sqrt{\frac{2 R_{c} \sqrt{\mathrm{Re}}}{D}}+22.2126\left[\operatorname{Re}\left(\frac{\mathrm{D}}{2 \mathrm{R}_{\mathrm{c}}}\right)^{2}\right]^{0.7888} \\
& \times \operatorname{Re}^{-0.71438}\left(\operatorname{Re}\left(\frac{\mathrm{D}}{2 \mathrm{R}_{\mathrm{c}}}\right)^{2}\right)^{-0.25}
\end{aligned}
$$

where $R_{c}$ is the internal bend radius. To determine the pressure drop in non $90^{\circ}$ bends the linear relation bellow is used:[14]

$$
\left(\frac{L_{e}}{D}\right)_{\beta}=\frac{\beta}{90 o}\left(\frac{L_{e}}{D}\right)_{90^{\circ}}
$$

The pressure losses encountered in three-pipe junctions can be predicted with the horizontal momentum equation. These losses are a function of the direction of the flow, the mass flow, and the size of the pipes. For a blowing piping system, as used for fluidic actuators, it is required to only determine the flow behavior in separating junctions. The pressure difference between the two pipes is given by [10]:

$$
\Delta p=\left(p_{\text {up }}-p_{\text {down }}\right)=\frac{1}{2} \rho u_{\text {up }}^{2}\left(K-1+q^{2} \psi^{2}\right)
$$

The value of $K$ depends on the flow types at the junction, which are schematically depicted in Fig. 7b. The coefficients $K_{\#}$ that correspond to Fig. $7 \mathrm{~b}$ are:

$$
\begin{aligned}
& K_{1}=1+q^{2} \psi^{2}-2 q \psi \cos \left[\frac{3}{4}(\pi-\theta)\right] \\
& K_{2}=\frac{1}{2}+q^{2} \psi^{2}-\frac{3}{2} q \psi \\
& K_{3}=1+\frac{q^{2}}{\psi^{2}}-2 \frac{q}{\psi} \cos \left[\frac{3}{4}(\pi-\theta)\right] \\
& K_{4}=1+\frac{q^{2}}{\psi^{2}}-2 \frac{q}{\psi} \cos \left(\frac{3}{4} \theta\right) \\
& K_{5}=\frac{1}{2}+q^{2} \psi^{2}-\frac{3}{2} q \psi \\
& K_{6}=1+q^{2} \psi^{2}-2 q \psi \cos \left(\frac{3}{4} \theta\right)
\end{aligned}
$$

where $q$ is the mass flow ratio $\left(\dot{m}_{\text {down }} / \dot{m}_{\text {up }}\right)$, with up representing the upstream pipe and down the downstream pipe. $\psi$ is the area ratio $\left(S_{\text {down }} / S_{\text {up }}\right)$. For example, for $K_{1}$ $q=\dot{m}_{B} / \dot{m}_{A}$ and $\psi=S_{B} / S_{A}$.

For this case study, the flow conditions downstream at the actuators are known, which means that at any junction the two downstream pipes have known conditions and the pressure in the upstream branch needs to be determined. The two downstream branches are used to determine the flow conditions in the upstream pipe using Eqs. (5) through (11). This might result in two different pressures found for the upstream branch. Clearly, this cannot be the case. Therefore, the downstream branch that yields the highest upstream pressure $\left(p_{\text {upmax }}\right)$ is kept the same while the branch that yields the lowest upstream pressure is fitted with an obstruction. This obstruction virtually changes the cross-sectional area of the pipe, thereby increasing the local pressure. The dimension of the obstruction is determined by first calculating the desired downstream pressure using Eqs. (5) through (11) with $p_{\text {up }_{\max }}$. Subsequently, the 
difference between the newly calculated downstream pressure and the original pressure is computed $\left(\Delta p_{m}\right)$. Finally, the new pipe area with the obstruction $\left(S_{h}\right)$ is found using the Borda-Carnot equation:

$$
\begin{aligned}
& \Delta p_{m}=\frac{\rho}{2} u^{2}\left(\frac{S_{s}}{\alpha S_{h}}-1\right)^{2} \\
& \alpha=0.6+0.4\left(\frac{S_{h}}{S_{s}}\right)^{2}
\end{aligned}
$$

where $S_{S}$ is the actual original pipe area.

Tapping bleed air from the compressor can lead to losses in thrust and specific fuel consumption. Therefore, an onboard pump can be used. In this case, the air compressor must be sized to determine the additional weight and power consumption that this subsystem adds to the complete PJA system. After the pneumatic analysis is complete, the mass flow $(\dot{m})$ and the pressure ratio $\left(p_{2} / p_{1}\right)$ between free stream air and the beginning of pneumatic line are known. The values expected for these two parameters can be typically delivered by a centrifugal compressor. The mass of a centrifugal compressor $\left(W_{\text {compressor }}\right)$ consists of the following components: the impeller mass $\left(W_{\text {imp }}\right)$, the diffuser mass $\left(W_{\text {dif }}\right)$, the casing mass $\left(W_{\text {cas }}\right)$ and the AC (alternating current) motor mass $\left(W_{\mathrm{ACmotor}}\right)$ :

$W_{\text {compressor }}=W_{\text {imp }}+W_{\text {dif }}+W_{\text {cas }}+W_{\text {ACmotor }}$

Centrifugal compressors can be sized through the use of two nondimensional parameters: the specific speed $(N s)$ and the specific diameter $(D s)$. The compressor efficiency ( $\left.\eta_{\text {compressor }}\right)$ is strictly related to the specific speed through an empirical relationship due to Gong et al. [15] To achieve a high efficiency, it is assumed that the compressor has a specific speed equal to 0.8 which leads to a compressor efficiency of 0.9 . Using the empirical relation between $N_{\mathrm{s}}$ and $D_{\mathrm{s}}$ for centrifugal compressors [15] and the assumption that $N_{\mathrm{s}}=0.8$, a specific diameter of approximately 5 is obtained. The actual diameter $(D)$ of the compressor's impeller can be estimated using the following equation [15]:

$D=\frac{\operatorname{Ds} \sqrt{\dot{v}}}{\left(g H_{\mathrm{ad}}\right)^{1 / 4}}$

where $\dot{v}$ is the volumetric air mass flow $\left[\mathrm{m}^{3} / \mathrm{s}\right], g$ the gravitational acceleration and $H_{\mathrm{ad}}$ is the adiabatic head:

$H_{\mathrm{ad}}=\frac{\gamma p_{1}}{\gamma-1}\left[\left(\frac{p_{2}}{p_{1}}\right)^{\frac{\gamma-1}{\gamma}}-1\right]$

with $\gamma$ being the heat capacity ratio ( $\gamma=1.4$ for air), $p_{1}$ the pressure at the compressor inlet and $p_{2}$ the pressure at the compressor outlet.
The weight of the impeller is determined using an empirical relationship between the impeller diameter and weight from $\mathrm{Xu}$ and Amano [16]. The weight of the diffuser and casing of the compressor are determined using a relation from Tornabene et al. [17] that applies for compressors with $N_{\mathrm{s}}=0.8$ and $p_{2} / p_{1}=3$; very similar conditions to the case study at hand. It relates the diffuser and casing mass as function of the mass flow (m).

Finally, the mass of the AC motor is determined $\left(W_{\mathrm{ACmotor}}\right)$. To do this, the power to be delivered by the motor $\left(P_{\text {motor }}[\mathrm{W}]\right)$ is required to be:

$P_{\text {motor }}=\frac{\dot{\mathrm{m}} L_{\mathrm{ad}}}{\eta_{\text {compressor }} \eta_{\text {shaft }} \eta_{\text {motor }}}$

where $\eta_{\text {compressor }}=0.9, \eta_{\text {shaft }}$ is assumed to be equal to $0.95, \eta_{\text {motor }}$ is assumed to be equal to 0.9 and $L_{\mathrm{ad}}$ is the adiabatic work that the compressor needs to perform:

$L_{\mathrm{ad}}=\frac{c_{p} T_{1}}{\gamma}\left[\left(\frac{p_{2}}{p_{1}}\right)^{\left(\frac{\gamma-1}{\gamma}\right)}-1\right]$

with $c_{p}$ being the specific heat of air (approximately 1.004 $\mathrm{kJ} / \mathrm{kg} / \mathrm{K}$ ) and $T_{1}$ being the compressor inlet temperature, which depends on the altitude at which the aircraft is flying. With $P_{\text {motor }}$ computed, the weight of the motor is obtained using the empirical relation between power and motor weight given by Bari et al. that holds for AC motors

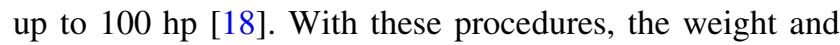
the power required by the centrifugal compressor is determined and accordingly accounted for in the PJA system.

\subsection{Electrical wiring interconnection system design method}

To ease the description of the method to design the electrical wiring interconnection system (EWIS), all power or data receiving components will be identified as sinks and all origins of power (e.g., a power center) and data signals (e.g., the communication centers) will be referred to as sources. Power and data signals travel from a source to a sink over custom wires with a certain resistivity and shielding (and therefore weight properties) for which selection criteria apply. These criteria have been captured in this research. Similar wires are bundled, requiring extra wrapping material with associated weight (also modeled), to form manufacturable components. These so-called wiring harnesses are bundles of electrical wires held together with cable ties, clamps or conduits (not modeled). A connector is assembled on each end of the wiring harness, so that harnesses can be easily attached to each other or to electrical aircraft components. 
Various harnesses are interconnected at the so-called production breaks that create a manageable manufacturing and assembly process, but also introduce some extra weight (modeled). Wiring harnesses may contain hundreds of wires, and provide connectivity between all the mission and vehicle systems. As they are critical to the mission, they need to ensure sufficient redundancy and reliability. The separation of wires or entire bundles is enforced by numerous opposing design rules and regulations, for example, redundancy of flight controls, electromagnetic compatibility or heat dissipation of power cables.

A custom software tool named CAESAR (Conceptual Analysis of EWIS System Architectures) has been developed to automate the conceptual design process for the EWIS. CAESAR is designed to generically model different system architectures and electrical system design options (e.g., data or power) and provide a quick estimate on EWIS weight, volume (space occupied) and cost. The CAESAR tool automatically generates a conceptual space reservation topology from the main pathway definition and system positioning information. From the geometric model, an undirected weighted graph representation is derived that serves as the basis for the signal routing process (see Fig. 8).
CAESAR has fully parametrized the EWIS design process. Some parameters are inputs provided by the user (or other software tool) and others are derived parameters in the sense that engineering rules define how to evaluate them from the inputs. The input for the initial space reservation mainly consist of 3D positioning of clients, sources and production breaks as well as connectivity information (i.e., how are the production break connected to each other). This is enough information for CAESAR to generate the space reservation geometry. The electrical signals are allowed to travel on a predefined grid. This grid is defined by the production breaks as depicted in Fig. 8 . These production breaks and their interconnections with each other can be specified though user inputs but also automatically generated in a similar fashion as for the pneumatic lines (described in Sect. 2, 2.1); it makes use of the geometry of the fuselage and wings together with a number of variables that will completely define their position.

In addition, the user specifies which sink is connected to which source, and what should be the redundancy $(N)$. If a power cable is specified between a source and a sink, the type (AC or DC), the voltage $(V)$, and the current $(I)$ need to be specified. If a communication cable is specified, the

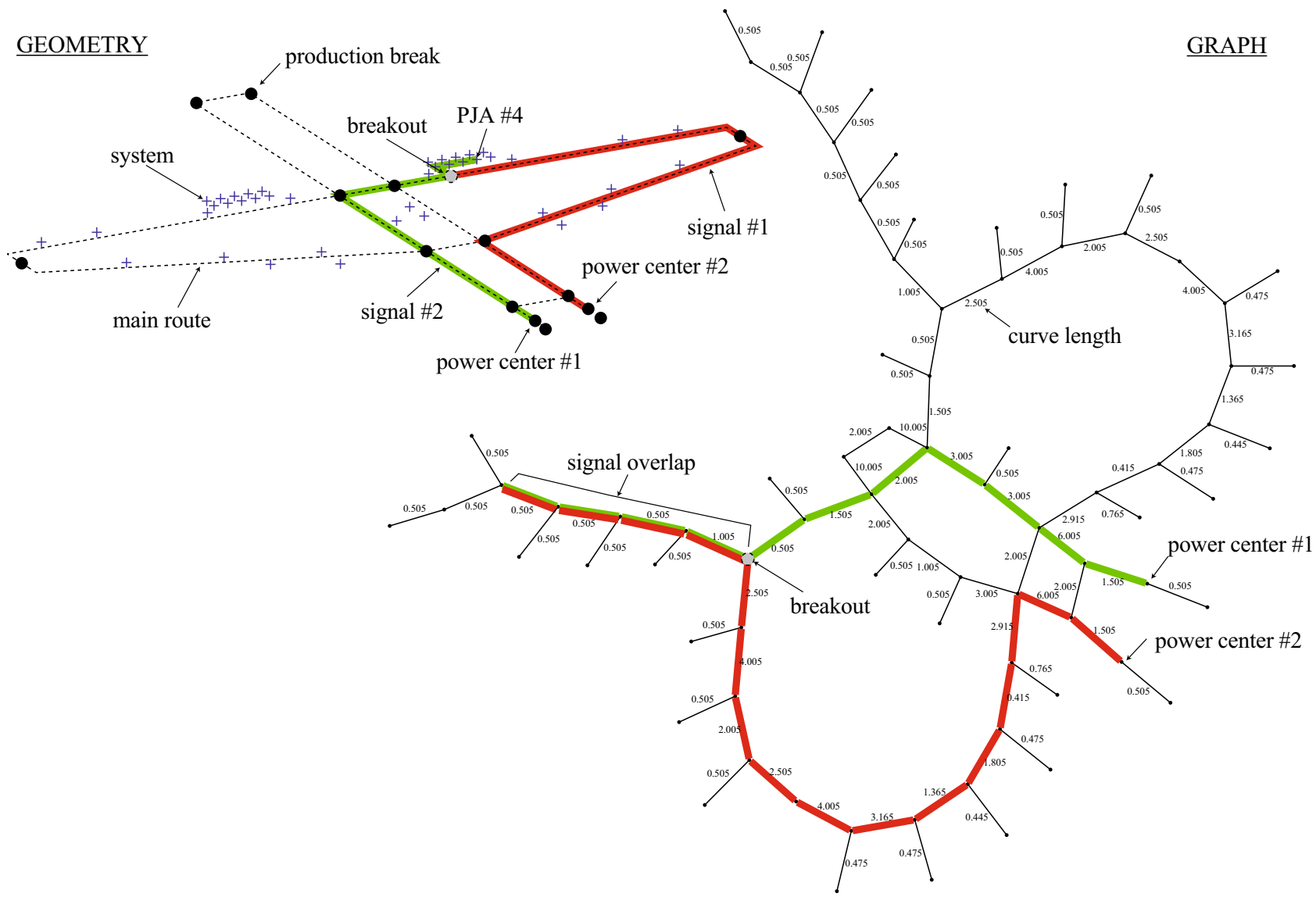

Fig. 8 Representation of EWIS main pathway definition. Left geometric model. Right corresponding undirected, weighted graph 
protocol (e.g., analog, ARINC429 or AFDX), the data rate, and maximum time delay need to be specified. In addition, it needs to be specified whether the cable is sensitive to electromagnetic interference (EMI). In collaboration with Fokker Elmo, a database has been established that contains a number of both aluminum and copper cables typically used to connect power systems in aerospace applications as well as a number of communication cables. The tool is able to consult the database and select the appropriate cable choice given the input requirements. The information stored per cable in the database includes the gauge, the mass per unit length $(\mathrm{m} / \mathrm{l})$, and the resistivity $(\omega)$.

Wire and bundle separation is an important aspect of the EWIS design process. The electromagnetic compatibility (EMC) can restrict the proximity of wires carrying incompatible signals - they cannot be in the same bundle, and bundles typically must be separated by a certain physical distance. A wire routing constraint might take the form If signal = AC Then do not route with flight controls. There is an EMC matrix that defines the actual relationships between signals that can travel together or not. These interrelations are defined as constraints. Four EMC classifications are used: HiDC for high-voltage DC wires, HiAC for high current $\mathrm{AC}$ wires, $\mathrm{M}$ for other wires, and $\mathrm{S}$ for wires that are susceptible to interference.

This research accounts for both segregation and separation of wires. Segregation is an aspect on aircraft level and involves the actual segregation of wires into separate bundles. In the context of PJA, segregation is an important means to ensure redundancy in system functionality by providing multiple signals to the same system that travel over distinct routes through the aircraft with a minimum overlap. This reduces the likelihood of total system failure when one of the cable/harnesses is compromised. Separation is an aspect on a bundle level and deals with minimum distance constraints between adjacent wires/bundles to ensure electromagnetic compatibility and proper heat dissipation characteristics. Adequate separation is a function of the physical and electrical attributes of the wires inside a bundle and the hazard potential of a failure at any given point along the length of the bundle.

To route a wire between a source and a sink, the shortest path is found using a weighted undirected graph where production breaks or harness breakouts become the nodes, and the edges are the harness segments between breaks. The weights of the edges are set equal to their physical length, an appropriate measure for cable weight, which needs to be minimized.

Most of the power and communication functionality should be provided with a certain level of redundancy, most often two or maximally three distinct signal paths should be followed. In this case, the routing problem becomes more challenging and there are no standard algorithms available to solve it. The extended formulation is:

$\min J=\left(\sum_{i}^{N} \sum_{j} l_{j}+\sum_{k} \alpha_{1} l_{k}+\sum_{l} \alpha_{2} V_{l}\right)$

The optimal routing of Eq. (19) introduces several terms and states that the optimal solution for the routing of $N$ redundant signals corresponds to the combination of paths that has minimum length $\left(\sum_{i}^{N} \sum_{j} l_{j}\right)$, but also minimum path overlap $\left(\sum_{k} l_{k}\right)$ and a minimum number of production break (vertex) intersections $\left(\sum_{l} V_{l}\right)$; aspects that act against each other. Equation (19) is a weighted multi-objective function where two weight factors $\alpha_{1}$ and $\alpha_{2}$ penalize path and vertex overlap relative to total path length, respectively. A complicating factor is introduced by the requirement to route redundant signals from different power/communication sources to further improve reliability. This transforms the routing problem into a multisource, single destination problem where every next redundant signal switches to an alternative source node. Figure 9 clearly shows the different routing results for one signal (a), two redundant signals (b) and three redundant signals (c) on an aircraft with two power centers. The same exact principle holds true for the communication network.

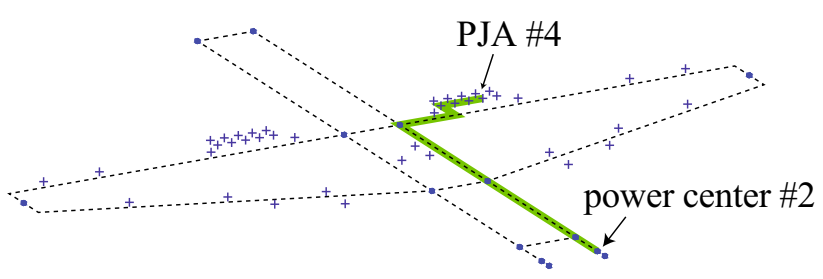

(a) Shortest path

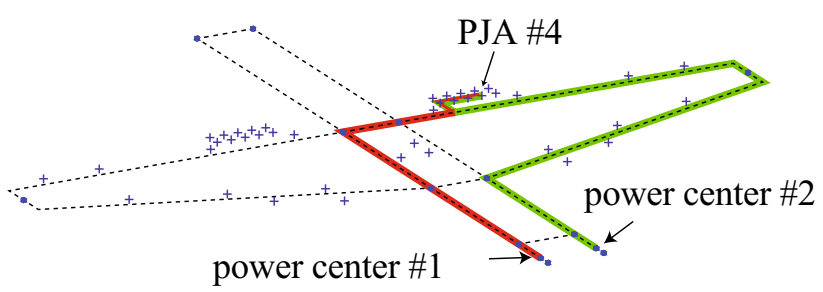

(b) Two redundant signals

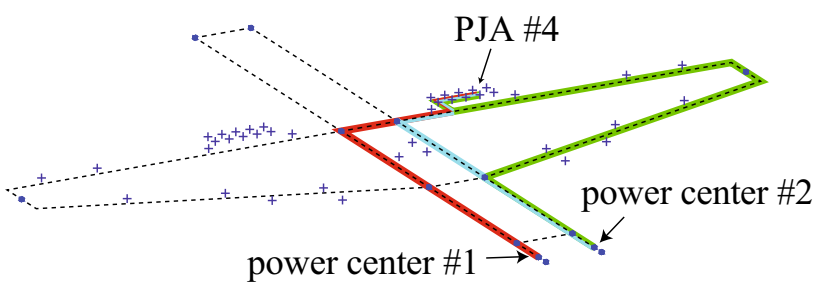

(c) Three redundant signals

Fig. 9 Example of result generated by automatic routing algorithm for a single client (PJA 4) with varying levels of redundancy 
An exhaustive search was implemented that finds all possible paths for all signals and then performs a combinatory study evaluating Eq. (19) on each combination of paths. This approach guarantees an optimal result, but is not efficient. However, the relatively low number of signals that need routing for this research and the relative acyclicness of the main pathway topology render the current approach feasible and adequate. Moreover, two assumptions are made. First, the criticality of overlap for different main pathway segments and production breaks is consistently the same. For this reason Eq. (19) uses $\alpha_{1}$ and $\alpha_{2}$ instead of $\alpha_{k}$ and $\alpha_{l}$ that would otherwise specialize on different pathways. Second, the objective is to completely favor minimum overlap over minimum length. In this respect, weight factors approach infinity $\alpha_{1} \rightarrow \infty$ and $\alpha_{2} \rightarrow$ $\infty$ and the routing lengths might be over-conservative. If there are no redundancy requirements, one signal travels from one source to one sink and the optimal routing corresponds to the shortest path (i.e., $\alpha_{1}=\alpha_{2}=0$ ).

The selection process for power or communication cables differs in complexity. Communication cables are typically quite specific and the selection process boils down to a direct relationship between communication protocol and cable type. For power cables there is a larger range of cables available and the selection process consists of two steps: the selection of material type and cable size. Power cables are either made from aluminum or copper cores. While the default choice is aluminum, some conditions would favor copper. This is true if the cable travels through an area with limited space, an area with high temperatures or an unprotected area and has a small gauge.

Evaluation of these rules for each cable involves detailed knowledge of the various zones in the aircraft. In the current stage of the research, high-level assumptions are made for the applicability of the former conditions. Once the type of metal is defined, the appropriate cable should be chosen from a cable database. ${ }^{2}$ Wires carrying current $(I)$ always have inherent resistance, or impedance, to current flow. The driving rule for power cable selection is that the cable should result in an acceptable voltage drop $(\Delta V)$, which is defined as the amount of voltage loss that occurs through all or part of a circuit due to impedance. The allowable voltage drop can be expressed as a relative constraint, such as $\Delta V_{\max } \leq 4 \% V$ ( $V$ is the source voltage), or an absolute value, such a $\Delta V_{\max } \leq 6.5$. The idea is to iterate through the database and select the cable with a combination of resistivity $(\omega)$ and nominal cross sections (a) that just satisfies the following constraint:

\footnotetext{
${ }^{2}$ Note that this rule is somewhat implicit, because the gauge can only be known once a cable is selected (using the rules below) An initial selection of a copper cable has to be performed first, after which a conditional second selection step for an aluminum cable could follow.
}

$$
\frac{\omega}{a} \leq \frac{\Delta V_{\max }}{I \cdot l}
$$

\subsection{Fuel burn estimations}

The required fuel mass to operate the electrical system and the pneumatic system is computed from the mechanical off-takes (MOT) and bleed air off-takes (BOT), respectively. The fuel mass due to bleed air off-takes $\left(W_{f_{\mathrm{BOT}}}\right)$ is computed using the following relationship [19]:

$$
W_{f_{\mathrm{BOT}}}=\frac{K_{B}\left(p_{3} / p_{2}\right)^{0.475} \dot{m}}{K_{E}}\left(\exp ^{\mathrm{t} \mathrm{K}_{\mathrm{E}}}-1\right)
$$

where $K_{B}$ is the bleed air power off-take factor which is assumed to be equal to 0.00499 [20], $p_{3} / p_{2}$ is the compressor pressure ratio assumed equal to 24.9 [19], and $t$ the time over which the system is used. To estimate the mechanical off-take fuel mass $\left(W_{f_{\mathrm{MOT}}}\right)$ the following is used [19]:

$$
W_{f_{\mathrm{MOT}}}=\frac{K_{P} P_{\mathrm{PJA}} W_{\text {avgaircraft }}}{n_{\text {engines }} T_{\mathrm{TO}}}\left(\exp ^{\mathrm{t} \mathrm{K}_{\mathrm{E}}}-1\right)
$$

where $K_{P}$ is the mechanical power off-take factor assumed to be $0.01163 \mathrm{~N} / \mathrm{W}$ [20], $W_{\text {avgaircraft }}$ is the average mass of the aircraft during the time in which the system is used, $n_{\text {engines }}$ is the number of engines, $T_{\mathrm{TO}}$ the take-off thrust of one engine and $K_{E}$ is an efficiency factor defined as follows:

$$
K_{E}=\operatorname{SFC}\left(\frac{\cos (\gamma)}{\mathrm{L} / \mathrm{D}}+\sin (\gamma)\right)
$$

with SFC being the specific fuel consumption, $\gamma$ the flight path angle and $L / D$ the lift-to-drag ratio. The system requirements (PPJA and $\dot{m}$ ) are the technology performance metrics (TPMs) that are derived from the system automatically designed system architectures.

\section{Verification of prediction results}

The methodology presented in Sect. 2 consists of a chain of analytical tools that are interconnected. Each analysis block outlined in Fig. 4 introduces errors with respect to a real aircraft with a detailed system design. Since there are no active PJA systems documented in the open literature with disclosed weight data, it is challenging to assess the accuracy of the predicted system weight. The EWIS is based on an industry database of actual wires, and the selection procedure was developed in cooperation with Fokker ELMO. The design procedure for wire routing and reliability has been invented by the authors as a logical means of best practice for wire routing in support of the 
PJA system. The mass and volume of the system electronics (DC converter, signal generator, HV-ASIC) as well as the PJAs themselves are based on best estimates from experts of the Fraunhofer institute.

Best practices have also been applied to the architecture of the ducting assembly. The duct sizing has been

Table 1 Inputs used for the validation study of the Initiator

\begin{tabular}{llll}
\hline & Unit & $\mathrm{A} 320-200$ & $\mathrm{~B} 737-800$ \\
\hline$N_{\text {pax }}$ & & 150 & 162 \\
$W_{\mathrm{p}}$ & $\mathrm{kg}$ & 20536 & 21,319 \\
$M_{\text {cruise }}$ & & 0.76 & 0.79 \\
$h_{\text {cruise }}$ & $\mathrm{m}$ & 11,278 & 11,887 \\
$R_{h}$ & $\mathrm{~km}$ & 2870 & 1363 \\
$L_{\text {take-off }}$ & $\mathrm{m}$ & 2180 & 2101 \\
$L_{\text {landing }}$ & $\mathrm{m}$ & 1440 & 1440 \\
$A$ & & 9.39 & 9.45 \\
$C_{L_{\text {max,landing }}}$ & & 3.2 & 3.4 \\
$C_{L_{\text {max,take-off }}}$ & & 2.2 & 2.2 \\
$C_{L_{\text {max }, \text { clean }}}$ & & 1.2 & 1.2 \\
\hline
\end{tabular}

generated based on mass flow requirements of the PJA system, while its route through the aircraft is based on the location of the engine (source), fuel tank, and primary structural components. The wall thickness for the ducts was obtained from a $1.8 \mathrm{~m}$ a physical piece of pneumatic ducting used in a Boeing 737. Furthermore, this ducting piece was also used to verify design rules and mass prediction. For example, the article showed that behind a junction the sum of the cross-sectional areas of the two ducts equalled the cross-sectional area of the single duct ahead of the junction. The increase in weight due welds, connections and other refinements has been taken into account using a correction factor on the predicted weight, which originally only accounted for length, diameter and wall thickness.

To demonstrate the overall accuracy of the Initiator design program, two aircraft were automatically synthesized for a set of top-level requirements taken from the A320-200 and 737-800, respectively. These midrange aircraft were chosen because their requirements were close to those that were used for the test aircraft (Sect. 4). The top-level requirements along with important input parameters are shown in Table 1 . These inputs
Table 2 Results of the validation study of the Initiator (version 2.3)
Table 3 Mission requirements and performance input at airplane level to be used in the Initiator

\begin{tabular}{|c|c|c|c|c|c|c|c|}
\hline & \multirow[t]{2}{*}{ Unit } & \multicolumn{3}{|c|}{ A320-200 } & \multicolumn{3}{|c|}{ B737-800 } \\
\hline & & Initiator & Literature & Error $(\%)$ & Initiator & Literature & Error $(\%)$ \\
\hline$T / W$ & & 0.286 & 0.31 & -8 & 0.31 & 0.31 & 0 \\
\hline$W / S$ & & 5445 & 5900 & -8 & 5360 & 5550 & -3 \\
\hline$C_{D_{0}}$ & cts & 213 & 230 & -7 & 223 & 240 & -7 \\
\hline Oswald factor, $e$ & & 0.75 & 0.76 & -1 & 0.73 & 0.71 & 3 \\
\hline$W_{\mathrm{OE}} / W_{\mathrm{TO}}$ & & 0.50 & 0.54 & -8 & 0.54 & 0.59 & -9 \\
\hline$W O E$ & tons & 33.3 & 39.7 & -16 & 34.5 & 41.4 & -17 \\
\hline$W_{\mathrm{TO}}$ & tons & 66.8 & 68 & -2 & 64.3 & 70.5 & -9 \\
\hline
\end{tabular}

\begin{tabular}{lll}
\hline Parameter & Symbol & Value \\
\hline Number of passengers & $N_{\text {pax }}$ & 180 \\
Payload mass & PM & $18,600 \mathrm{~kg}$ \\
Cruise Mach number & $M_{\text {cruise }}$ & 0.78 \\
Cruise altitude & $h_{\text {cruise }}$ & $11,280 \mathrm{~m}$ \\
Range & $R_{\text {ac }}$ & $2960 \mathrm{~km}$ \\
Take-off distance & $l_{\text {take-off }}$ & $1960 \mathrm{~m}$ \\
Landing distance & $l_{\text {landing }}$ & $1490 \mathrm{~m}$ \\
Airworthiness regulations & & FAR-25 \\
Loiter time & $t_{\text {loiter }}$ & $30 \mathrm{~min}$ \\
Maximum lift-to-drag ratio & $(L / D)_{\text {max }}$ & 17 \\
Specific fuel consumption & SFC & $0.6 \mathrm{~N} / \mathrm{Nhr}$ \\
Maximum landing lift coefficient & $C_{L_{\text {max,landing }}}$ & From 2.0 to 3.0 in steps of 0.1 \\
Maximum take-off lift coefficient & $C_{L_{\text {max,ake-off }}}$ & 2.0 \\
\hline
\end{tabular}


Table 4 Input parameters for piping assembly

\begin{tabular}{|c|c|c|}
\hline Input & Value & Unit \\
\hline $2 b_{\text {fairing }- \text { in }} / b_{\text {wing }}$ & 0.28 & \\
\hline $2 b_{\text {fairing-out }} / b_{\text {wing }}$ & 0.5 & \\
\hline$c_{\mathrm{LE}} / c_{\text {wing }}$ & 0.2 & \\
\hline$c_{\mathrm{TE}} c_{\text {wing }}$ & 0.84 & \\
\hline$o_{\mathrm{LE}} / t_{\text {wing }}$ & 0.5 & \\
\hline$o_{\mathrm{TE}} / t_{\mathrm{wing}}$ & 0.5 & \\
\hline$c_{\text {actuator-in }} / c_{\text {flap-in }}$ & 0.5 & \\
\hline$c_{\text {actuator-out }} / c_{\text {flap-out }}$ & 0.5 & \\
\hline$b_{\text {actuator-in }} / b_{\text {flap-in }}$ & 0.9 & \\
\hline$b_{\text {actuator-out }} / b_{\text {flap-out }}$ & 0.9 & \\
\hline$c_{\text {pipe-in }} / c_{\text {flap-in }}$ & 0.2 & \\
\hline$c_{\text {pipe-out }} / c_{\text {flap-out }}$ & 0.2 & \\
\hline$o_{\text {pipe-in }} / t_{\text {flap-in }}$ & 0.5 & \\
\hline$o_{\text {pipe-out }} / t_{\text {flap-out }}$ & 0.5 & \\
\hline$o_{\text {fuselage }} / \varnothing_{\text {fus }}$ & 0.05 & \\
\hline$o_{\text {floor }} / \varnothing_{\text {fus }}$ & 0.05 & \\
\hline$\rho_{\text {pipe }}$ & 2710 & $\mathrm{~kg} / \mathrm{m}^{3}$ \\
\hline$t_{\text {pipe }}$ & 1 & $\mathrm{~mm}$ \\
\hline$d_{\text {actuator }}$ & 10 & $\mathrm{~mm}$ \\
\hline$\theta_{\text {bend }}$ & 90 & $\circ$ \\
\hline
\end{tabular}

See Fig. 5 for definition of dimensions

were collected from sources in the open literature [22, 23].

In Table 2 the results of the Initiator are compared to results reported in the open literature [22, 23]. It can be seen that the maximum take-off mass for the A320 and B737 is predicted within 2 and $9 \%$, respectively. Although this is an acceptable accuracy from a conceptual design point of view, it should be noticed that the various contributions that influence the maximum take-off weight (i.e., $\left.C_{D_{0}}, e, W_{\mathrm{OE}}\right)$ are underestimated up to $17 \%$. Later versions of the Initiator (i.e., version 2.5) already demonstrate great improvements in the individual contributions as is shown by Elmendorp et al. [9]. However, the present version of the Initiator is deemed accurate enough to show the effect of pulsed jet actuators on the change in aircraft mass and fuel consumption for a given set of top-level requirements and the design process of the Initiator.

\section{Test case definition}

The method described above is applied to a test case with similar top-level requirements of a typical mid-range aircraft. The PJAs are assumed to be functioning whenever the flaps of the aircraft are deflected. This means at the beginning of the mission during take-off and the initial part of climb and at the end of the mission during final descent and touch down. A simplified mission profile is assumed without a diversion phase. The mission requirements and the performance parameters are listed in Table 3. The maximum lift coefficient attainable during landing $\left(C_{L_{\text {max,landing }}}\right)$ is assumed to be taking a range of values from 2.0 to 3.0 in steps of 0.1 . It is assumed that the aircraft has slats, even at the lowest assumed lift coefficient. This is to account for the uncertainty in performance of PJAs. For each value of $C_{L_{\text {max,landing }}}$ the tool is run. Provided that the landing distance requirement is actively constraining the wing size (which is the case), for each different value of $C_{L_{\text {max,landing }}}$ a different aircraft design is obtained.

All three configurations depicted in Fig. 6 are run in addition to a baseline configuration without the PJA system on-board. Furthermore, for each of the three PJA configurations, two sets of runs are made to investigate the effect of having "reliability 1 " or "reliability 2" for the EWIS design:

- Reliability 1: each client is connected to the two power centers and two communication centers by means of 1 wire, so the total number of wires per client is 4 .

- Reliability 2: each client is connected to the two power centers and two communication centers by means of 2 wires, so the total number of wires per client is 8 .

The result yields $(3 \times 2+1) \times 11=77$ design iterations that have been carried out in approximately three nominal working days on a PC workstation.

Table 4 lists the exact system inputs that were used to design the piping assembly. These inputs are the same for all cases. All the values are expressed in percentage with respect to either the wing chord $\left(c_{\text {wing }}\right)$, wing thickness $\left(t_{\text {wing }}\right)$, half wing span $\left(b_{\text {wing }} / 2\right)$, flap chords $\left(c_{\text {flap }}\right)$, flap spans $\left(b_{\text {flap }}\right)$ and flap thicknesses $\left(t_{\text {flap }}\right)$.

\section{Results and discussion}

All 77 designs were conceived using the methodology presented in Sect. 2 and shown to converge within $1 \%$ of the maximum take-off weight and within $0.2 \%$ within the desired mission range. Four key performance indicators (KPIs) were identified: maximum take-off mass, operating empty mass, fuel mass, and payload-range efficiency. Each of these KPIs is plotted against the assumed maximum lift coefficient in Fig. 10. One should interpret these graphs as follows: the increase in maximum lift coefficient affects the size of the wing, i.e., the larger $C_{L_{\max }}$ the smaller the wing. This has a "snowball" effect on all of the KPIs, i.e., it lowers the operating empty mass, the fuel mass, and the take-off mass and increases the payload-range efficiency. 


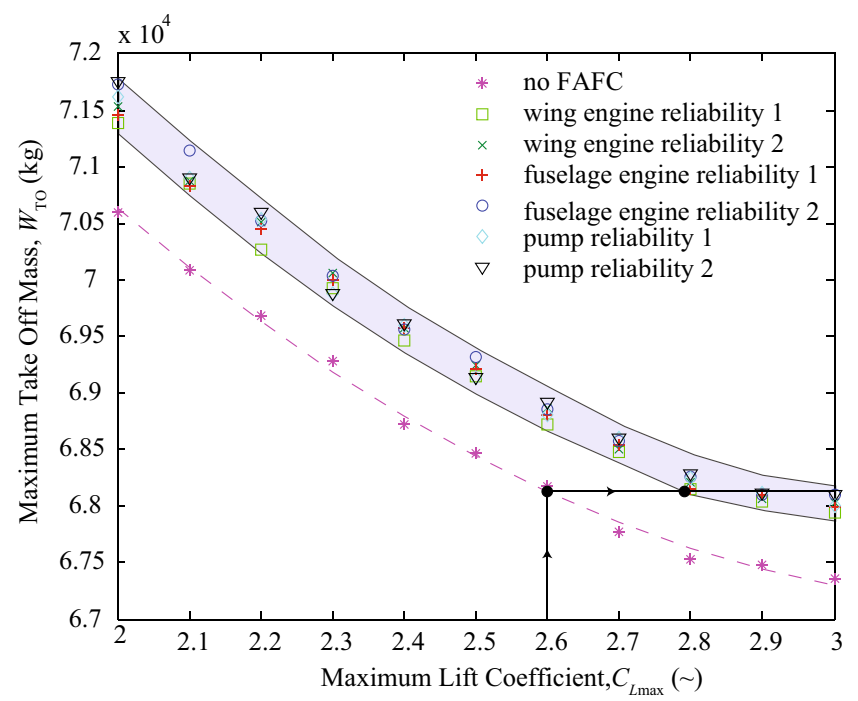

(a) Maximum take-off weight

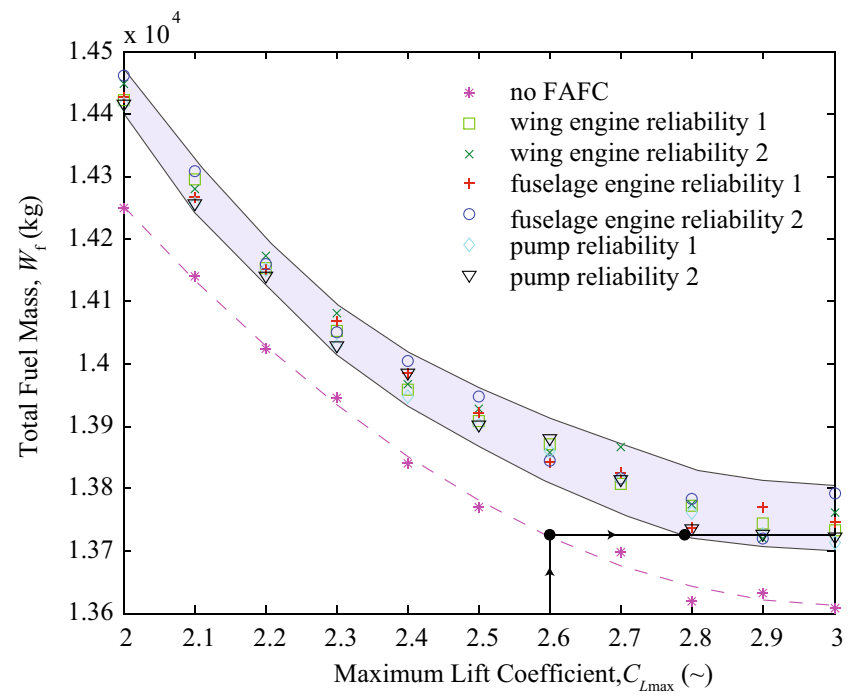

(c) Total fuel weight

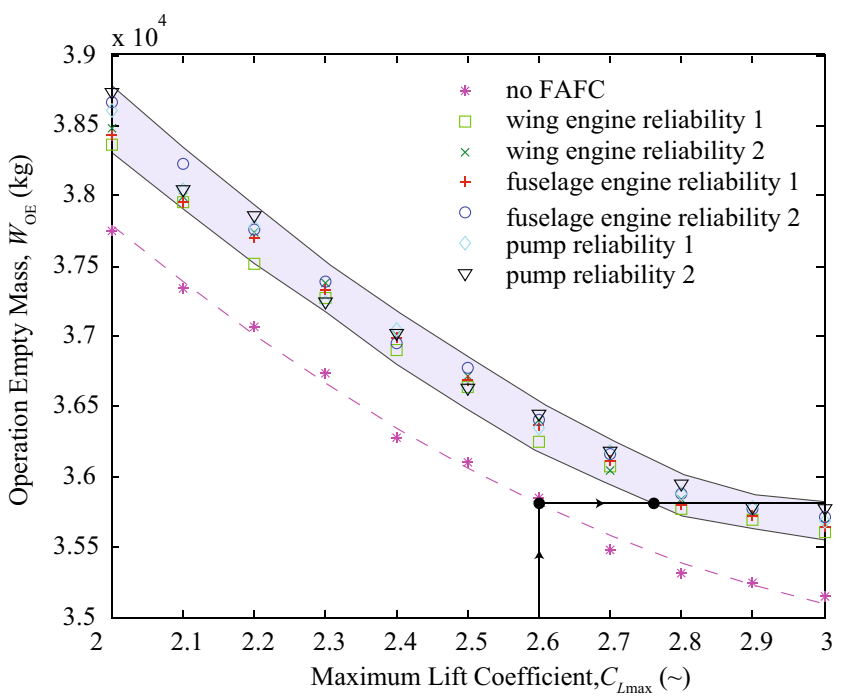

(b) Operative empty weight

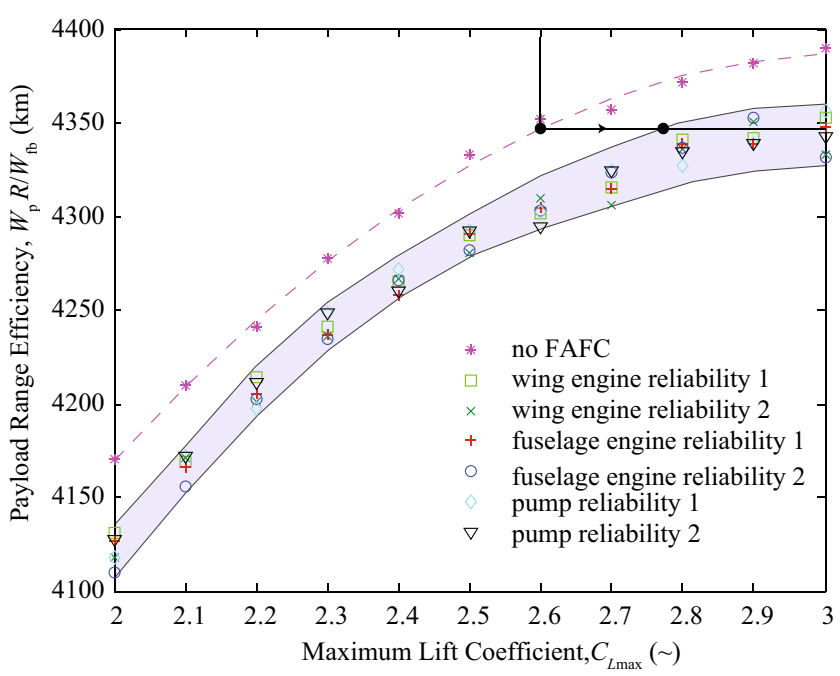

(d) Payload-range efficiency

Fig. 10 Effect of PJA-induced increase in maximum lift coefficient on key performance indicators

The increase in $C_{L_{\max }}$ can be due to the installation of the PJAs.

The dashed line in each graph represents the change in each KPI with maximum lift coefficient without the addition of the pulsed jet actuators. We see that with increasing maximum lift coefficient, the fuel mass and maximum take-off mass decrease as we expect for this design that is constrained by its landing distance. Within the gray band, we see designs incorporating various system architectures and aircraft configurations. We see that they follow a similar trend as the baseline design but that for a given maximum lift coefficient they have worse KPIs than the baseline design without the system on-board. Naturally, this difference is explained by the increase in system mass and power consumption that has a negative effect on all performance indicators.

Inside the gray band, several combinations of configuration and reliability are plotted. No clear trend can be deduces as to the effect of reliability on the key performance indicators. It is thought that the convergence criteria ( $1 \%$ of MTOM and $0.2 \%$ error in mission range) might have been too crude to see a consistent difference between the two reliability cases. It can be stated that the effect of reliability on the KPIs is fairly small. This can also be concluded for the configuration (wing-mounted engines, fuselage-mounted engines, or electrical pump). There is no consistent effect of this on the KPIs over the entire range of maximum lift coefficients that has been investigated here. 
It can be seen, however, that in eight out of the eleven cases that were investigated, the pump configuration yielded the lowest amount of fuel burn. This seems to point towards a positive effect on fuel burn of a more electric aircraft architecture compared to an architecture based on engine bleed air. On the other hand, we also see a weight penalty in the operating empty mass (OEM) with the pump configuration yielding the highest OEM in eight out of the eleven cases.

The black line with arrows shows what improvement in maximum lift coefficient is required to "break even" in terms of the four KPIs. For example, if the baseline aircraft can achieve a $C_{L_{\max }}$ of 2.6, its maximum take-off mass equals 68.1 metric tons. When the PJA system is fully installed, it causes a weight penalty of 0.7 metric tons in terms of MTOM and 0.5 metric tons in terms of OEM. To offset this weight penalty and keep the maximum take-off mass constant, the PJAs need to increase the maximum lift coefficient to at least 2.8. It can be deduced from the black lines in each of the plots of Fig. 10 that a typical increase of $C_{L_{\max }}$ on the order of $0.2-0.4$ is required to justify the added weight and power off-takes of the engine. Assuming an increase in $C_{L_{\max }}$ of 0.4 can indeed be achieved, this would reduce the maximum take-off mass by approximately 0.2 metric tons $(-0.3 \%)$, reduce the operating empty mass by 0.2 metric tons $(-0.6 \%)$, and cause the total fuel mass to stay the same. Finally, the payload-range efficiency would increase by approximately $15 \mathrm{~km}$ $(+0.3 \%)$.

Based on the theoretically estimated [7] (two-dimensional) $c_{l_{\max }}$ increase of 0.7 , it seems plausible that PJAs can indeed cause an increase in $3 \mathrm{D} C_{L_{\max }}$ of approximately 0.4. However, experimental studies should still confirm that this can actually be achieved for a 3D wing with Fowler flap. Apart from this uncertainty, the improvements in the key performance indicators are relatively mild. Moreover, the additional complexity that the integration of this system introduces in the aircraft (particularly in the Fowler flap) has not been taken into account in this study. The added complexity yields higher development and manufacturing cost. In addition, maintenance cost have to increase due to the fact that the PJAs become part of the flight critical high-lift system, and are therefore subjected to the same reliability requirements as the high-lift mechanism that controls the deployment of flaps and slats.

\section{Conclusions}

A study has been carried out to investigate the effect of flap-based pulsed jet actuators on the key performance indicators of a mid-range jet aircraft. Automated design software was successfully used to design the required piping assembly and electrical wiring interconnection system that supports the operation of the pulsed jet actuators. Assuming a maximum lift coefficient of 2.6 in landing configuration, it was shown that the addition of the pulsed jet system causes an increase in operating empty mass from 55.8 metric tons to 56.3 metric tons for an A320-like aircraft. This increase could only be offset if a maximum lift coefficient increase of at least 0.2 was achieved by the pulsed jet actuators. In addition, it was demonstrated that when the maximum lift coefficient increases to 0.4 due to the pulsed jet actuators only a mild decrease in operating empty mass $(-0.6 \%)$ and maximum take-off mass $(-0.3 \%)$ can be expected due to the smaller wing that is then required. The total fuel burn is expected to stay the same due to the additional fuel that is required to power the system when the flaps are deployed.

Acknowledgments The authors would like to acknowledge the valuable contribution of Fokker Elmo with respect to the design of the EWIS system. In addition, Martin Schüller and Martin Lipowski from Fraunhofer ENAS for providing details on the actuator specifications. This research was conducted within the Smart Fixed Wing Aircraft (SFWA) framework as part of the Clean Sky 1 program.

Open Access This article is distributed under the terms of the Creative Commons Attribution 4.0 International License (http://crea tivecommons.org/licenses/by/4.0/), which permits unrestricted use, distribution, and reproduction in any medium, provided you give appropriate credit to the original author(s) and the source, provide a link to the Creative Commons license, and indicate if changes were made.

\section{References}

1. Pugliese, A.J., Englar, R.J.: Flight testing the circulation control wing. Presented at the AIAA aircraft system technology meeting, AIAA 1979-1791, New York (1979)

2. Kibens, V., Bower, W.W.: An overview of active flow control applications at the Boeing company. In: Proceedings of the 2nd AIAA Flow Control Conference, pp. 2004-2624 (2004)

3. Greska, B., Krothapalli, A., Seiner, J.M., Jansen, B., Ukeiley, L.: The effects of microjet injection on an F404 jet engine. In: Proceedings of the 11th AIAA/CEAS Aeroacoustics Conference, AIAA 2005-3047, Monterery, CA, 23-25 May 2005

4. Seiner, J., Ukeiley, L., Jansen, B.: Aero-performance efficient noise reduction for the F404-400 Engine. In: Proceedings of the 11th AIAA/CEAS Aeroacoustics Conference, AIAA 2005-3048, Monterery, CA, 23-25 May 2005

5. Shaw, L., Smith, B., Saddoughi, S.: Full-Scale flight demonstration of active control of a pod wake. In: Proceedings of the 3rd AIAA Flow Control Conference, San Francisco, CA, 5-8 June 2006

6. Cattafesta, L., Sheplak, M.: Actuators for active flow control. Ann. Rev. Fluid Mech. 43, 247-272 (2011)

7. Shmilovich, A., Yadlin, Y.: J. Active flow control for practical high-lift systems. J. Aircr. 46(4), 1354 (2009)

8. Schueller, M., Lipowski, M.: Actuators for Active Flow Control. Cleansky Internal Meeting Presentation, Fraunhofer (2013) 
9. Elmendorp, R.J. M., Vos, R., LaRocca, G.: A conceptual design and analysis method for conventional and unconventional aircraft. In: Grant, I. (ed.) Proceedings of the 29th Congress of the International Council for the Aeronautical Sciences, ICAS, St. Petersburg, Russia (2014)

10. Bassett, M., Winterbone, D., Pearson, R.: Calculation of steady flow pressure loss coefficients for pipe junctions. In: Proceedings of the Institution of Mechanical Engineers, Part C, Journal of Mechanical Engineering Science, vol. 215(8), pp. 861-881 (2001)

11. Barber, A.: Pneumatic Handbook, 7th edn. The Trade \& Technical Press Limited, Surrey (1989)

12. Anon.: Engineering Toolbox, Roughness \& Surface Coefficients of Ventilation Ducts (Online; Accessed April 2013). http://www. engineeringtoolbox.com/surface-roughness-ventilation-ducts-d_ 209.html

13. Crawford, N., Cunningham, G., Spedding, P.L.: Prediction of pressure drop for turbulent fluid flow in 90-degree bends. In: Proceedings of the Institution of Mechanical Engineers, Part E, Journal of Mechanical Engineering, vol. 217, pp. 153-155 (2003)

14. Crane, C.: Flow of fluids through valves, fittings, and pipes. Tech. rep., Crane Co, Joliet, IL (1988) (Technical Paper 410)

15. Gong, Y., Carstens, N., Driscoll, M., Matthews, I.: Analysis of radial compressor options for supercritical co2 power conversion cycles. Tech. rep., MIT Department of Nuclear Science and Engineering (2006) (Report No. MIT-GFR-034)
16. Xu, C., Amano, R.S.: Empirical design considerations for industrial centrifugal compressors. Int. J. Rotat. Mach. 2012, 1 (2012)

17. Tornabene, R., Wnag, X., Steffen, C.J., Freeh, J.: Development of parametric mass and volume models for an aerospace sofc/gas turbine hybrid system. Tech. rep., National Aeronautics and Space Administration (2005) (NASA TM-2055-213819)

18. Bari, M., Roof, C., Oza, A., Chudobay, B.: The future of electric aircraft. In: Proceedings of the 51st AIAA Aerospace Sciences Meeting, Grapevine, TX, 7-10 Jan 2013

19. Scholz, D.: Betriebskostenschaetzung von flugzeugsystemen als beitrag zur entwurfsoptimierung. Deutscher luft- und raumfahrtkongress, Bonn, Germany, 26-29 Sept 1995

20. Ahlefelder, S.: Kraftstoffverbrauch durch entnahme von zapfluft und wellenleistung von strahltriebwerken. Master's thesis, Hamburg University of Applied Sciences (2006)

21. Jenkinson, L., Simpkin, P., Rhodes, D.: Civil Jet Aircraft Design. Butterworth-Heinemann, Oxford (1999)

22. Roux, E.: Avions civils à reaction, Plan 3 vues et données caractéristiques, 3rd edn. Elodie Roux (2007)

23. Obert, E.: Aerodynamic Design of Transport Aircraft. IOS press, Delft (2009) 